\title{
Effects of Dialkyldimethylammonium Bromides on the Membrane Properties of Cationic Liposomes
}

\author{
Akiko Inagaki $^{1}$, Koji Tsuchiya ${ }^{1}$, Hideki SAKaI ${ }^{1,2}$, Tomohiro Imura ${ }^{1}$, Takahiro OHKubo ${ }^{1}$, \\ Nobuyuki Tsubaki ${ }^{3}$, Shoko Yoкоуама ${ }^{4 *}$ and Masahiko AbE ${ }^{1,2}$ \\ ${ }^{I}$ Faculty of Science and Technology, Tokyo Universtity of Science \\ (2641 Yamazaki, Noda, Chiba 278-8510, JAPAN) \\ ${ }^{2}$ Institute of Colloid and Interface Science, Tokyo University of Science \\ (1-3 Kagurazaka, Shinjuku, Tokyo 162-0825, JAPAN) \\ ${ }^{3}$ Oleochemicals Research Lab., NOF Co. \\ (Amagasaki, Hyogo 660-0095, JAPAN) \\ ${ }^{4}$ School of Pharmaceutical Sciences, Kyushu University of Health and Welfare \\ (1714-1 Yoshino-cho, Nobeoka-city, Miyazaki 882-8508, JAPAN)
}

Edited by S. Ozeki, Shinsyu Univ., and accepted March 25, 2005 (received for review January 11, 2005)

\begin{abstract}
Cationic liposomes composed of dipalmitoylphosphatidylcholine (DPPC) and dialkyldimethylammonium bromides (DLAB, DMAB, DPAB or DSAB) were prepared by the Bangham method, and the effect of the alkyl chain length of dialkyldimethylammonium bromide on the membrane properties of the liposomes was examined in terms of the trapping efficiency, liposomal shape and phase transition temperature. Furthermore, the effect of stearylamine (SA) on the liposomal membrane properties was also investigated. Large unilamellar vesicles (LUVs) with a diameter of approximately $1.0 \sim 1.5 \mu \mathrm{m}$ were formed by mixing small amounts of cationic substances, not only dialkyldimethylammonium bromides, but also SA. The trapping efficiency of the mixed liposomes was greater than that of the DPPC liposomes since the DPPC liposomes were multilamellar vesicles. The maximum trapping efficiency of the mixed liposomes was obtained at 0.05 mole fractions of the cationic substances. Furthermore, dialkyldimethylammmonium bromides with longer alkyl chains, DSAB, DPAB and DMAB, formed cationic substance-rich liposomes with DPPC, and they had the second maximum trapping efficiency at $0.75 \sim 0.9$ mole fractions of cationic substance.
\end{abstract}

Key words: large unilamellar vesicle, dialkyldimethylammonium bromide, cationic liposome, freeze-fracture electron microscopy, stearylamine

\section{Introduction}

Liposomes are drug carriers that are both biodegradable and of minimal toxicity, and liposomal drug delivery systems have been widely researched for the purpose of targeting drugs to specific cells (1). Recently, cationic liposomes have been anticipated to be potential drug carriers for gene therapy (2-4) from the viewpoint of their complex association between the negatively charged DNA and the cationic liposomes. Large unilamellar vesicles (LUVs) are useful as drug carriers due to their high trapping efficiency. Many techniques, such as the organic solvent injection method (5), the reverse phase evaporation (RPE) method (6) and the supercritical reverse phase evaporation (scRPE) method (7) have been reported for the preparation of liposomes.

\footnotetext{
*Correspondence to: Shoko Yokoyama, School of Pharmaceutical Sciences, Kyushu University of Health and Welfare, 1714-1 Yoshino-cho, Nobeoka-city, Miyazaki 882-8508, JAPAN

E-mail: s.yokoyama@phoenix.ac.jp
} 
Liposomes prepared by the Bangham method (8) are generally multilamellar vesicles (MLVs). We found in the previous paper (9) that LUVs having a high trapping efficiency were easily obtained by mixing a small amount $\left(X_{\mathrm{DPAB}}=0.05\right)$ of a cationic amphiphilic substance, dipalmityldimethylammonium bromide (DPAB), with dipalmitoylphosphatidylcholine (DPPC) in the common Bangham method. There is no necessity for the use of supercritical carbon dioxide, large amounts of organic solvents such as ether and methanol, or a specific apparatus. Cationic liposomes, such as mixed DPPC/DPAB liposomes, are expected to be useful as drug carriers, although the safety of cationic amphiphilic substances requires further study. In addition, the preparation method of the mixed DPPC/DPAB liposomes will aid in the preparation of LUVs.

In this study, cationic liposomes composed of DPPC and dialkyldimethylammonium bromides (DAAB) with various alkyl chains were prepared, and the effect of alkyl chain length of DAAB on the membrane properties of the liposomes was investigated.

\section{Experimental}

\subsection{Materials}

Dilauryldimethylammonium bromide (DLAB), dimyristyldimethylammonium bromide (DMAB), dipalmityldimethylammonium bromide (DPAB) and distearyldimethylammonium bromide (DSAB) were purchased from Tokyo Kasei Kogyo Co. Ltd. Stearylamine (SA) and D-glucose were purchased from Sigma Co. and Wako Pure Chemical Ind., Ltd., respectively. L- $\alpha$-Dipalmitoylphosphatidylcholine (DPPC) was supplied by NOF Co. Water for injection (Ohtsuka Pharmaceutical Co.) was used as the solvent.

\section{$\mathbf{2} \cdot 2$ Preparation of Cationic Liposomes}

Mixed DPPC/DAAB liposomes and mixed DPPC/SA liposomes were prepared according to the procedure by the Bangham method (8); DPPC and (DAAB or SA) were dissolved in chloroform in a test tube; the solvent was then removed by blowing nitrogen gas into the test tube, and the residual solvent was further dried overnight at room temperature in a desiccator under vacuum; a $4 \mathrm{ml}$ portion of water was added to this lipid film and warmed $\left(60^{\circ} \mathrm{C}\right)$ above the phase transition temperature of DPPC for $10 \mathrm{~min}$; the test tube was then shaken vigorously on a vortex mixer, and mixed $\mathrm{DPPC} /(\mathrm{DAAB}$ or SA) liposomes were obtained.

\subsection{Measurement of Trapping Efficiency of Liposomes}

Glucose was trapped into the liposomes. Briefly, glucose was dissolved in water, liposomes were prepared using the glucose solution instead of water as described in Section 2.2. The concentrations of liposomes and glucose were 5 and $0.2 \mathrm{mM}$, respectively, under consideration of osmotic pressure. The liposome dispersion was dialyzed against $0.1 \mathrm{M} \mathrm{NaCl}$ solution using a cellulose tube (Viskase Companies, Inc.) to remove the nonencapsulated glucose remaining in the dispersion medium. The temperature was kept at $5^{\circ} \mathrm{C}$ during the dialysis. The liposomes inside the tube were then destroyed by addition of ethanol. The amount of glucose in the solution was determined by the mutarotase GOD method (10) using a spectrophotometer (MPS-2000, Shimadzu Co.).

The trapping efficiency was calculated according to the equation.

Trapping efficiency $(\%)=\left(C_{\text {in }} / C_{\text {total }}\right) \times 100$ where $C_{\text {in }}$ and $C_{\text {total }}$ are the amount of glucose trapped in liposomes and the total glucose in the system, respectively.

\section{2-4 Freeze-Fracture Electron Microscopy}

Liposome sample was frozen immediately in liquid propane. The frozen sample was fractured in a freezereplica apparatus (FR-7000A, Hitachi Science Co.) at $-150^{\circ} \mathrm{C}$. Replica was made by platinum-carbon shadowing followed by platinum shadowing. It was cleaned by acetone and distilled water, and then picked up on 150 mesh copper grids and observed by an electron microscope (JEM-1200EX, Japan Electron Co.). The outline was shown in our previous paper (11).

\subsection{Measurement of Phase Transition Temperature of Liposomal Bilayer Membranes}

The phase transition temperature between gel and liquid crystalline states of liposomal bilayer membranes was measured with a differential scanning calorimeter (DSC 8230, Rigaku Denki Co.). Liposome solution was put in a sampling vessel, which is made of stainless steel, and then the vessel was sealed. The measurement conditions were $1 \mathrm{~K} / \mathrm{min}$ for the scanning rate, 20 
$80^{\circ} \mathrm{C}$ for the scanning range and $0.1 \mathrm{~m} \mathrm{cal} / \mathrm{s}$ for the sensitivity. The total lipid concentration was $20 \mathrm{mM}$.

\section{Results and Discussion}

\section{$3 \cdot 1$ Trapping Efficiency of Liposomes}

The trapping efficiencies of the liposomes were measured using an aqueous-space marker, glucose, and the results are shown in Fig. 1.

As can be seen in Fig. 1, the trapping efficiency of the DPPC liposomes remarkably increased by mixing a small amount of DAAB in the DPPC liposomes. One maximum value was observed at 0.05 mole fractions of DAAB. Furthermore, an additional maximum value was observed at a higher mole fraction of DAAB, except for the mixed DPPC/DLAB system: the values of $X_{\mathrm{DAAB}}$ were $0.75,0.75$ and 0.90 for the DPPC/DMAB, DPPC/DPAB and DPPC/DSAB liposomes, respectively. Namely, cationic amphiphilic substances having longer alkyl chains formed cationic amphiphilic substance-rich liposomes.

It is known that the trapping efficiency of LUVs is larger than that of MLVs, and that the trapping efficiency increases with the increasing particle size of the liposomes (7). Therefore, the shape of the liposomes was observed, as reported in the next section, to clarify the increase in the trapping efficiency of liposomes due to the inclusion of DAAB,

\section{$3 \cdot 2$ Freeze-Fracture Electron Micrographs of Liposomes}

The electron micrographs of the freeze-fracture repli-

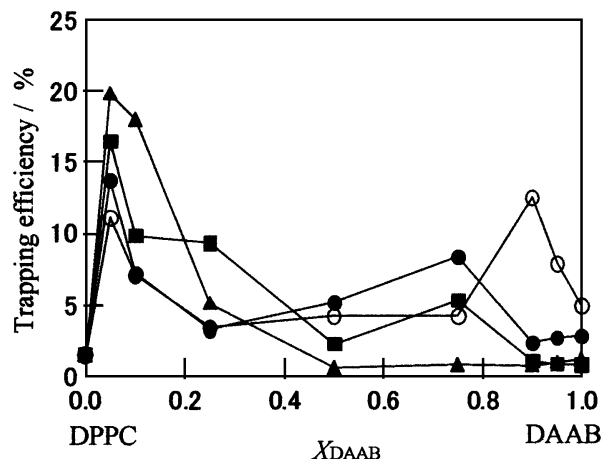

Fig. 1 Trapping Efficiency of DPPC/DAAB Liposomes.

DAAB: $\boldsymbol{\Lambda}, \mathrm{DLAB} ; \mathbf{\square}, \mathrm{DMAB} ; \boldsymbol{O}, \mathrm{DPAB}$

$\bigcirc$, DSAB.Total lipid concentration: $5 \mathrm{mM}$. cas of the DPPC/DLAB $\left(X_{\mathrm{DLAB}}=0.05\right), \mathrm{DPPC} / \mathrm{DMAB}$ $\left(X_{\mathrm{DMAB}}=0.05\right), \mathrm{DPPC} / \mathrm{DSAB}\left(X_{\mathrm{DSAB}}=0.05\right), \mathrm{DPPC} /$ $\operatorname{DSAB}\left(X_{\mathrm{DSAB}}=0.9\right)$ and DPPC/DMAB $\left(X_{\mathrm{DMAB}}=0.75\right)$ mixed liposomes, which were observed to have the maximum trapping efficiency, are shown in Fig. 2.

As can be seen in Fig. 2, the mixed DPPC/DAAB liposomes were LUVs with a diameter of approximately $1.0 \sim 1.5 \mu \mathrm{m}$. The electron micrographs of the freezefracture replicas of the DPPC/DPAB liposomes $\left(X_{\mathrm{DPAB}}=\right.$ 0.05 and 0.75 ) were shown in our previous paper (9), and the shape of the liposomes was that of LUVs. Whereas the shape of the DPPC liposomes is that of MLVs.

The fracture faces of the DPPC/DLAB liposomes $\left(X_{\mathrm{DLAB}}=0.05\right)$ and the DPPC/DMAB liposomes $\left(X_{\mathrm{DMAB}}=\right.$ $0.05)$ exhibited banded texture, which corresponds to the $\mathrm{P}_{\beta}$ ' phase (11) caused by the quenching temperature. Thus the structure of the DPPC/DLAB and
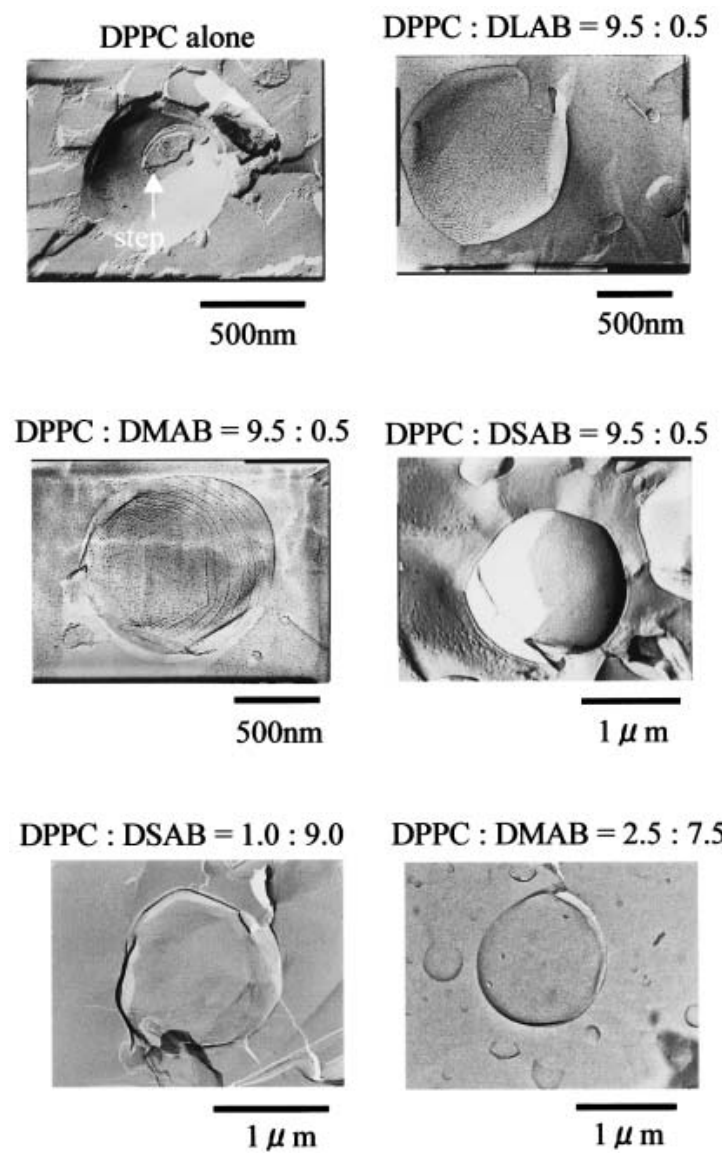

Fig. 2 Freeze-Fracture Electron Micrographs of Liposomes. Total lipid concentration: $20 \mathrm{mM}$. 
DPPC/DMAB liposomes is not that of MLVs but LUVs.

The high trapping efficiencies of the mixed DPPC/DAAB liposomes were confirmed to be due to the liposomal shape, that of LUVs. It was found in the previous paper (9) that the shape of the mixed DPPC/DPAB liposomes $\left(X_{\mathrm{DPAB}}=0.25\right)$, which had the minimum trapping efficiency, was that of somewhat smaller LUVs with a diameter of approximately 500 $\mathrm{nm}$. Therefore, the shape of the mixed DPPC/DAAB liposomes is considered to be that of LUVs, although the ability of liposome formation in the DPPC/DLAB system decreased at higher mole fractions of DLAB.

\section{$3 \cdot 3$ Effect of DAAB on the Phase Transition Temperature of Liposomal Bilayer Membranes}

The phase transition temperatures of the mixed DPPC/DAAB liposomes from the gel to the liquid crystalline states were measured by DSC, and the results are shown in Fig. 3.

The phase transition temperature of the DPPC liposomes was shifted to a higher temperature by mixing it with DSAB or DPAB, and the mixed DPPC/DSAB liposomes $\left(X_{\mathrm{DPAB}}=0.3 \sim 0.75\right)$ and the mixed DPPC/ DPAB $\left(X_{\mathrm{DPAB}}=0.25 \sim 0.4\right)$ had the maximum phase transition temperature. The higher phase transition temperature implies a tight packing state of the liposomal bilayer membranes and cooperative interaction among the DPPC and DAAB. The cooperative interaction among the DPPC and DSAB molecules became largest when the mole fractions of DSAB were $0.3 \sim$ 0.75 . The trapping efficiency of the DPPC/DSAB lipo-

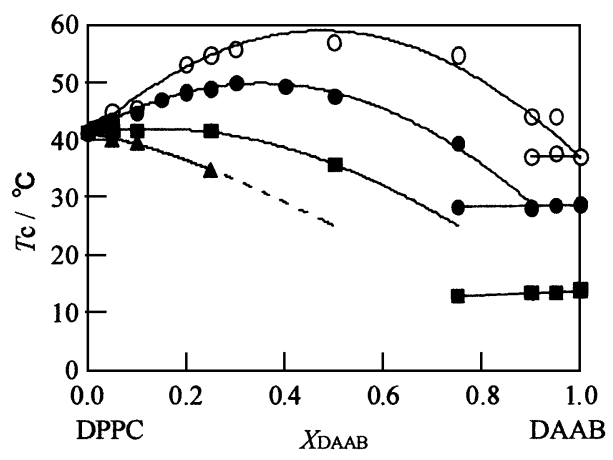

Fig. 3 Phase Transition Temperature of Liposomes. Symbols are the same as in Fig. 1. Total lipid concentration: $20 \mathrm{mM}$. somes $\left(X_{\mathrm{DSAB}}=0.25 \sim 0.75\right)$ was low, as shown in Fig. 1. These facts are explained as follows: DPPC and DSAB molecules are most densely packed in the liposomal bilayer membranes and the curvature of the liposomal particle was resultantly large. It was confirmed in the previous paper (9) that the shape of the mixed DPPC/DPAB liposomes $\left(X_{\mathrm{DPAB}}=0.25\right)$ having the highest transition temperature was that of the relatively smaller LUVs, with a diameter of approximately 500 $\mathrm{nm}$.

On the other hand, the phase transition temperature of the DPPC liposomes was shifted to a lower temperature by mixing it with DLAB. At $0.05 \sim 0.25$ mole fractions of DLAB, the DSC peak of the DPPC/DLAB liposomes became broad compared with that of DPPC liposomes. Above 0.5 mole fractions of DLAB, it was difficult to find a clear peak, suggesting a decrease in the ability to form liposomes.

The order of the phase transition temperatures of DAAB was DSAB $>$ DPAB $>$ DMAB $>$ DLAB, indicating that the packing state of the bilayer membranes became tighter as the alkyl chain length of DAAB increased. Therefore, DSAB formed pure DSAB liposomes that had a higher trapping efficiency compared with the other DAAB. In addition, the hydrophobicity of DSAB is close to that of DPPC. Thus, DSAB-rich liposomes were easily formed and had a high trapping efficiency. The shape of the mixed DPPC/DSAB liposomes $\left(X_{\mathrm{DSAB}}=0.9\right)$ was that of LUVs as shown in Fig. 2, and the shape of the DSAB liposomes was that of MLVs, as were the DPAB liposomes (9).

The relationship between the phase transition temperature of DAAB and the trapping efficiency of the

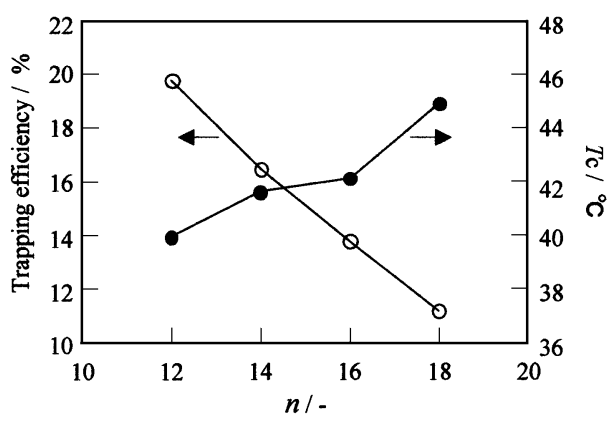

Fig. 4 Effect of Alkyl Chain Length of DAAB on Trapping Efficiency $(O)$ and Phase Transition Temperature (O) of the Mixed DPPC/DAAB Liposomes $\left(X_{\mathrm{DAAB}}=0.05\right)$. 
mixed DPPC/DAAB liposomes $\left(X_{\mathrm{DAAB}}=0.05\right)$ is shown in Fig. 4.

The trapping efficiency of the mixed DPPC/DAAB liposomes $\left(X_{\mathrm{DAAB}}=0.05\right)$ decreased with the increase of the alkyl chain length $(n)$ of the constituent DAAB and with the increase of the phase transition temperature of DAAB. Namely, the cooperative interaction among the DPPC and DAAB molecules became larger with increasing $n$ and resultantly the curvature of the liposomal particle was large. The order of trapping efficiencies of the mixed DPPC/DAAB liposomes $\left(X_{\mathrm{DAAB}}=\right.$ $0.05)$ is, therefore, explained by the decrease in the liposomal particle size (as the mean value).

\subsection{Effect of SA on the Trapping Efficien- cy of Liposomes}

The effect of SA, which has a mono alkyl chain, on the trapping efficiency of liposomes was examined, and the results are shown in Fig. 5. In Fig. 5, the data for the mixed DPPC/DSAB liposomes are also presented for the comparison between SA and DSAB.

As can be seen in Fig. 5, the mixed DPPC/SA liposomes $\left(X_{\mathrm{SA}}=0.05 \sim 0.1\right)$ had a high trapping efficiency, similar to the mixed DPPC/DSAB liposomes. The freeze-fracture electron micrograph of the DPPC/SA liposomes indicated that the shape of the DPPC/SA liposomes $\left(X_{\mathrm{SA}}=0.05 \sim 0.1\right)$ was that of LUVs with a diameter of approximately $1 \mu \mathrm{m}$.

Thus, LUVs are formed by mixing a small amount of a cationic substance with DPPC, not only cationic amphiphilic substances with dialkyl chains, but also cationic surfactants with monoalkyl chains. However,

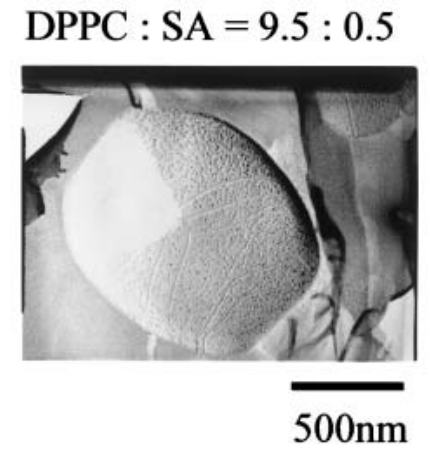

Fig. 6 Freeze-Fracture Electron Micrograph of the Mixed DPPC/SA Liposomes $\left(X_{\mathrm{SA}}=0.05\right)$.

the ability to form liposomes decreased when a large amount of SA was mixed with DPPC, whereas DSAB can form DSAB-rich liposomes. These facts are caused by their physicochemical properties: DSAB forms liposomes, while SA forms micelles (12) above the Kraft point.

Figure 7 shows the DSC curves for the DPPC/SA liposomes $\left(X_{\mathrm{SA}}=0 \sim 0.25\right)$.

DPPC liposomes showed a sharp endothermic peak. The endothermic peak shifted to a higher temperature and became broad as the mole fraction of SA increased. It is known that the DSC peak of LUVs is broad compared with that of MLVs (13). The DSC data shown in Fig. 7 indicate that the shape of the DPPC/SA liposomes $\left(X_{\mathrm{SA}}=0.05\right.$ and 0.1$)$ is that of LUVs. Above 0.25 mole fractions of SA, it was difficult to find a clear peak, indicating a decrease in ability of liposome formation at higher mole fractions of SA.

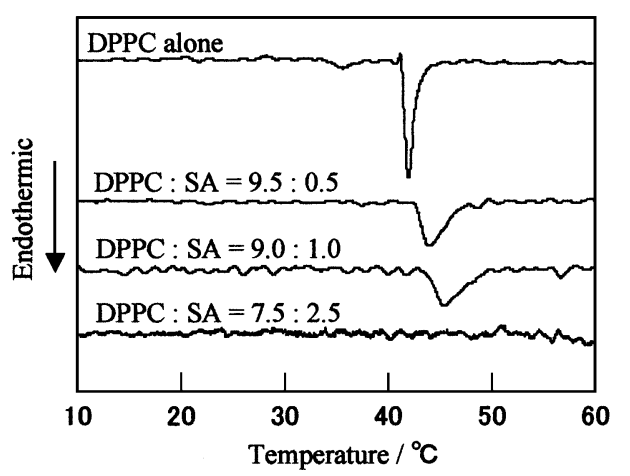

Fig. 7 DSC Curves for Mixed DPPC/SA Liposomes $\left(X_{\mathrm{SA}}=0 \sim 0.25\right)$.

Total lipid concentration: $20 \mathrm{mM}$. Efficiency of Cationic Liposomes. Total lipid concentration: $5 \mathrm{mM}$. 
Finally, we repeatedly state that the shape of the mixed DPPC/SA liposomes is that of LUVs. Many investigators (14-19) have been under the impression that the shape of the mixed DPPC/SA liposomes prepared by the Bangham method is that of MLVs, and they have never confirmed the liposomal shape. We emphasize that the shape of the DPPC/SA liposomes prepared by the Bangham method is that of LUVs and that the shape of the DPPC liposomes changes from that of MLVs to LUVs by the mixing of a small amount of SA into the DPPC liposomes.

\section{Conclusion}

The trapping efficiency of DPPC liposomes was greatly increased by mixing a small amount of DAAB. The maximum trapping efficiency of the mixed DPPC/DAAB liposomes was observed at 0.05 mole fractions of DAAB. The shape of the cationic liposomes was that of LUVs. DSAB, DPAB and DMAB also formed cationic substance-rich liposomes with DPPC, and they had the second maximum trapping efficiency at $0.75 \sim 0.9$ mole fractions of DAAB. Furthermore, it was found that LUVs were formed by mixing a small amount of SA with DPPC liposomes.

\section{References}

1. G. GREGORIADIS, Liposomes as Drug Carriers, Recent Trend and Progress, Wiley, Chichester (1988).

2. A. CONGIU, D. POZZI, C. ESPOSITO, C. CASTELLANO and G. MOSSA, Correlation between Structure and Transfection Efficiency: A Study of DC-Chol - DOPE/DNA Complexes, Colloids Surf. B: Biointerf., Vol. 36, 43-48 (2004).

3. L. CIANI, S. RISTORI, A. SALVATI, L. CALAMAI and G. MARTINI, DOTAP/DOPE and DC-Chol/DOPE Liposomes for Gene Delivery: Zeta Potential Measurements and Electron Spin Resonance Spectra, Biochim. Biophys. Acta, Vol. 1664, 70-79 (2004).

4. D. LLERES, J. M. WEIBEL, D. HEISSLER, G. ZUBER, G. DUPORTAIL and Y. MELY, Dependence of the Cellular Internalization and Transfection Efficiency on the Structure and Physicochemical Properties of Cationic Detergent/DNA/Liposomes, J. Gene Medicine, Vol. 6, 415-428 (2004).

5. S. BATZRI and E.D. KORN, Single Bilayer Liposomes Prepared Without Sonication, Biochim. Biophys. Acta, Vol. 298, 1015-1019 (1973).

6. F. Jr. SZOKA and D. PAPAHADJOPOULOS, Procedure for Preparation of Liposomes with Large Internal Aqueous Space and High Capture by Reverse-Phase Evaporation, Proc. Natl. Acad. Sci. U. S. A., Vol. 75, 4194-4198 (1978).

7. K. OTAKE, T. IMURA, H. SAKAI and M. ABE, Development of a New Preparation Method of Liposomes Using Supercritical Carbon Dioxide, Langmuir, Vol. 17, 3898-3901 (2001).

8. A.D. BANGHAM, M.M. STANDISH and J.C. WATKINS, Diffusion of Univalent Ions Across the Lamellae of Swollen Phospholipids, J. Mol. Biol., Vol. 13, 238-252 (1965).

9. S. YOKOYAMA, A. INAGAKI, T. IMURA, T. OHKUBO, N. TSUBAKI, H. SAKAI and M. ABE, Membrane Properties of Cationic Liposomes Composed of Dipalmitoylphosphatidylcholine and Dipalmityldimethylammonium Bromide, Colloids Surf. B: Biointerf., accepted.

10. I. MIWA, J. OKUDA, K. MAEDA and G. OKUDA, Mutarotase Effect on Calorimetric Determination of Blood Glucose with $\beta$ D-Glucose Oxidase, Clin. Chim. Acta, Vol. 37, 538-540 (1972).

11. K. HASHIZAKI, C. ITOH, H. SAKAI, S. YOKOYAMA, H. TAGUCHI, Y. SAITO, N. OGAWA and M. ABE, Freeze-Fracture Electron Microscopic and Calorimetric Studies on Microscopic States of Surface-Modified Liposomes with Poly(Ethylene Glycol) Chains, Colloids Surf. B: Biointerf., Vol. 17, 275282 (2000).

12. A.W. RALSTON, D.N. EGGENBERGER and F.K. BROOME, The Effect of Inorganic Electrolytes upon the Conductivity of Aqueous Solutions of Dodecylammonium Chloride, J. Am. Chem. Soc., Vol. 71, 2145-2149 (1949).

13. Y. TAKAICHI, S. KITTAKA and M. KODAMA, Calorimetric Studies of Phase Transition of Sonicated Vesicles of Dimyristoylphosphatidylcholine and Their Stabilities, Netsu Sokutei, Vol. 19, 103-112 (1992).

14. H. HOJO, Y. HOSHINO, T. KURITA and Y. HASHIMOTO, Modulation of Reticuloendothelian Activity with Liposomes, Res. Commun. Chem. Pathol. Pharmacol., Vol. 47, 373-385 (1985)

15. A. TABAK, E. HOFFER and U. TAITELMAN, Evaluation of a Liposome System for the Delivery of Desferrioxamine to Lungs in Rats, J. Pharm. Pharmacol., Vol. 46, $789-796$ (1993).

16. A. PANICO. R. PIGNATELLO and G. PUGLISI, Effects of Liposome Composition on Erythromycin Encapsulation and in Vitro Antibacterial Activity, Inter. J. Pharm. Adv., Vol. 1, 443458 (1996).

17. A. PANICO, R. PIGNATELLO, V. CARDILE and G. PUGLISI, Preparation of Liposome Formations Containing Immunomodulatory Peptides, Pharm. Acta Helve., Vol. 72, 1-10 (1997).

18. G.V. BETAGERI, N.B. VUTLA and A.K. BANGA, Liposomal Formation and Characterization of the Opioid Peptide Leucine Enkephalin, Pharm. Sci., Vol. 3, 587-591 (1997).

19. T. NAKANISHI, J. KUNISAWA, A. HAYASHI, Y. TSUTSUMI, T. HAYAKAWA and T. MAYUMI, Cationization of Liposomal Surface Charge Enhances Adjuvant Effect of Liposomes for Tumor Vaccine, Yakuzaigaku, Vol. 58, 59-68 (1998). 